\title{
Importancia del fomento de la lactancia materna
}

Drs. L. Marín, C. Márquez, P. Morales, B. Nuñez, B. Ortúzar.

La sustitución de la alimentación natural por leches artificiales, en las últimas décadas, viene inquietando desde algunos años a todos los que trabajan en el campo de la pediatría. Como ha sido señalado por diversos autores, la supresión precoz de la lactancia materna es especialmente nociva para los grupos socioeconómicos bajos, constituyendo un importante factor de riesgo de Desnutrición en el menor de un año, al quedar éste expuesto a una alimentación altamente contaminada y generalmente insuficiente.

Para combatir la precoz introducción de alimentación artificial favorecida por la publicidad, es necesario impactar a la población con las bondades de la lactancia materna. Con este fin, desde 1977 todo el equipo de salud del Consultorio Pudahuel toma conciencia de su papel en la labor educativa en pro de la lactancia materna y es así como la embarazada es informada sobre alimentación natural tanto por Matrona, Odontólogo, Nutricionista y Médico. Posteriormente la madre del R. N. es impulsada a alimentar a su hijo con pecho exclusivo hasta los cuatro meses, mediante charlas y controles de enfermería, y a su vez el pediatra refuerza esta actitud. Además si una madre en lactancia solicita Planificación Familiar, se le insta al uso de DIU, que no interfiere en la lactogénesis.

Este estudio está conducido a medir el impacto que la educación ha tenido sobre el tipo de alimentación láctea en el menor de un año y su influencia en el estado nutritivo de esta población. Se puede considerar que el tipo de alimentación es la única variable en juego entre 1976 y 1977 , ya que permanecen prácticamente constan-

Consultorio Pudahuel, Hospital San Juan de Dios, Area Occidente, Región Metropolitana. tes el nivel de desempleo, el analfabetismo, la higiene ambiental y otros factores de riesgos de desnutrición.

\section{PROPOSITO}

a) Cuantificar el impacto de la educación en la actitud de las madres con respecto a la lactancia materna.

b) Investigar si la lactancia materna disminuye la desnutrición en los menores de un año.

\section{METODOS}

Se realiza una auditoría de fichas clínicas y tarjetas de prestación de los nacidos en 1976 y 1977 , se escogen estos años por haber sido regidos por normas de atención pediátricas diferentes; en 1977 se comienza con el fomento de la lactancia materna a través de educación programada a madres de menores de un año y embarazadas. En cada año se toma aproximadamente un $50 \%$ de los nacidos vivos (485 en 1976 y 519 en 1977). Se analiza el estado nutritivo a $\operatorname{los} 3,4,8$ y 12 meses, meses en los cuales es habitual el registro de controles.

Para evaluar estado nutritivo se tomó la Tabla de Sempe (Peso-Edad).

\section{RESULTADOS}

Los resultados se expresan en porcentajes niñomes, dado que la muestra se analiza en cuatro edades distintas, por lo tanto la población total o $100 \%$ es el cuádruple de la muestra, así para 1976, en que se toman 485 casos, el cuádruple es 1.940; en 1977 se toman 519 casos, siendo el cuádruple de 2.076 .

Se toma un nivel de significación estadística del $5 \%$. 
Año 1976: 485 casos

\begin{tabular}{|c|c|c|c|c|c|c|c|c|c|c|c|c|c|c|c|c|}
\hline \multirow{2}{*}{$\begin{array}{l}\text { Edad } \\
\text { en } m .\end{array}$} & \multicolumn{5}{|c|}{ Aliment. Matema } & \multicolumn{5}{|c|}{ Aliment. Mixta } & \multicolumn{4}{|c|}{ Aliment. Artificial } & \multicolumn{2}{|r|}{ TOTAL } \\
\hline & $\mathbf{E}$ & Di & $\mathrm{D} 2$ & D3 & Subtot. & $\mathbf{E}$ & DI & $\mathrm{D} 2$ & D3 & Subtot. & $\mathrm{E}$ & DI & $\mathrm{D} 2$ & D3 & Subtot. & \\
\hline 3 & 211 & 6 & 1 & - & 218 & 118 & 11 & 3 & - & 132 & 112 & 17 & 6 & - & 135 & 485 \\
\hline 4 & 168 & 3 & 1 & - & 172 & 109 & 13 & - & 1 & 123 & 153 & 30 & 7 & - & 190 & 485 \\
\hline 8 & 90 & 1 & - & - & 91 & 66 & 9 & 2 & - & 77 & 254 & 49 & 9 & 5 & 317 & 485 \\
\hline 12 & 47 & 一 & - & - & 47 & 33 & 5 & 1 & - & 39 & 325 & 58 & 11 & 5 & 399 & 485 \\
\hline То'. & 516 & 10 & 2 & - & 528 & 326 & 38 & 6 & 1 & 371 & 844 & 154 & 33 & 10 & 1.041 & 1.940 \\
\hline
\end{tabular}

Año 1977: 519 casos

\begin{tabular}{|c|c|c|c|c|c|c|c|c|c|c|c|c|c|c|c|c|}
\hline \multirow{2}{*}{$\begin{array}{l}\text { Edad } \\
\text { en } m .\end{array}$} & \multicolumn{5}{|c|}{ Aliment. Materna } & \multicolumn{5}{|c|}{ Aliment. Mixta } & \multicolumn{4}{|c|}{ Aliment. Artificial } & \multicolumn{2}{|r|}{ TOTAL } \\
\hline & $\mathbf{E}$ & D1 & $\mathrm{D} 2$ & D3 & Subtot. & $\mathbf{E}$ & DI & $\mathrm{D} 2$ & D3 & Subtot. & $\mathrm{E}$ & D1 & $\mathrm{D} 2$ & D3 & Subtot. & \\
\hline 3 & 318 & - & - & - & 318 & 101 & 7 & 5 & 1 & 114 & 75 & 9 & 1 & 2 & 87 & 519 \\
\hline 4 & 283 & 2 & - & - & 285 & 95 & 10 & 2 & 1 & 108 & 106 & 14 & 2 & 4 & 126 & 519 \\
\hline 8 & 196 & 3 & 1 & - & 200 & 81 & 13 & 1 & - & 95 & 182 & 30) & 9 & 3 & 224 & 519 \\
\hline 12 & 116 & 10 & 1 & - & 127 & 74 & 19 & - & - & 93 & 239 & 49 & 7 & 4 & 299 & 519 \\
\hline TOT. & 913 & 15 & 2 & - & 930 & $35 \mathrm{I}$ & 49 & 8 & 2 & 410 & 602 & I02 & I9 & 13 & 736 & 2.076 \\
\hline
\end{tabular}

La tabla muestra los resultados de la observación de 485 casos en 1976 y 519 casos en 1977 a los 3,4 , 8 y 12 meses. Se muestra su distribución por tipo de alimentación láctea (natural, mixta, artificial) y estado nutritivo (eutrofia y desnutrición por grados).

En cuanto a alimentación, se observa que el tercer mes predomina la alimentación natural en ambos años, pero en 1977 es marcadamente mayor; así en 1976 tenemos 218 LM, 132 Mix. y 135 Art. y en 1977 hay 318 LM, 114 Mix. y 87 Art. A los 4 meses persiste el predominio de lactancia materna en 1977, en cambio en 1976 pasa a predominar la alimentación artificial; así en 1976 tenemos 172 LM, 123 Mix. y 190 Art.; en 1977 hay 285 LM, 108 Mix. y 126 Art. A los 8 meses hay un predominio de alimentación artificial en amibos años, siendo éste categórico en 1976 y escaso en 1977; para 1976 tenemos 91 LM, 77 Mix., 317 Art., y en 1977 hay 200 LM, 95 Mix. y 224 Art. A los doce meses se observa que el número de niños con lactancia materna y mixta es pequeño en 1976; en cambio, aunque en 1977 predomina la alimentación artificial, el número de niños con lactancia materna y mixta es aún alto. Tenemos que en 1976 hay 47 LM, 39 Mix., 399
Art. y en 1977 hay 127 LM, 93 Mix. y 299 Art.

Los desnutridos se distribuyen según tipo de alimentación en una configuración semejante en 1976 y 1977. Es importante el predominio de niños alimentados en forma artificial dentro del universo de desnutridos. En 1976 se observan 197 casos con alimentación artificial y sólo doce casos de desnutridos con LM. En forma similar en 1977 hay 134 desnutridos con alimentación artificial y sólo 17 con alimentación natural. Conviene resaltar que no hay desnutridos graves dentro de la población de niños alimentados al pecho, en ambos años; también es notorio el pequeño número de desnutridos graves con alimentación mixta; 1 caso en 1976 y 2 casos 1977 ; en comparación con 10 casos de D3 en 1976 y 13 en 1977 alimentados en forma artificial.

Cabe destacar que en el grupo con LM a los 12 meses a mayor población con LM aparecen casos de desnutrición; así en 1976 hay 47 niños con LM y ningún caso de desnutrición y en 1977 de 127 niños con LM hay 11 desnutridos.

En grupo alimentación artificial la distrofia aumenta progresivamente con la edad; en 1976 existen 23 desnutridos al tercer mes, 37 al cuarto, 63 al octavo y 74 a los 12 meses. En 1977 se 
observa una distribución semejante, encontrándose 12 desnutridos al tercer mes, al cuarto, 42 al octavo y 60 a los 12 meses.

La Tabla 1 muestra la distribución de la muestra según tipo de alimentación, englobando cada tipo tanto eutróficos como desnutridos; se observa un aumento de la lactancia de $27,2 \%$ (1976) a 44,7\% (1977).

Tabla 1

\begin{tabular}{|l|c|c|c|c|}
\cline { 2 - 5 } \multicolumn{1}{c|}{} & \multicolumn{2}{c|}{1976} & \multicolumn{2}{c|}{1977} \\
\cline { 2 - 5 } & N. ${ }^{\circ}$ Total & $\%$ & N. $^{\circ}$ Total & $\%$ \\
\hline $\begin{array}{l}\text { Alimentación } \\
\text { Materna }\end{array}$ & 528 & 27,20 & 930 & $\mathbf{4 4 , 7 0}$ \\
\hline $\begin{array}{l}\text { Alimentación } \\
\text { Artificial }\end{array}$ & 1.041 & 53,65 & 736 & 35,48 \\
\hline $\begin{array}{l}\text { Alimentación } \\
\text { Mixta }\end{array}$ & 371 & 19,20 & 410 & 19,74 \\
\hline TOTAL & 1.940 & 100,00 & 2.076 & 100,00 \\
\hline
\end{tabular}

La Tabla 2 es la división de la población estudiada en eutróficos y desnutridos, por año.
Se observa que la desnutrición de $13,09 \%$ en 1976 disminuye a $10,11 \%$ en 1977 .

Tabla 2

\begin{tabular}{|l|r|r||r|r|}
\cline { 2 - 5 } \multicolumn{1}{c|}{} & \multicolumn{2}{c||}{1976} & \multicolumn{2}{c|}{1977} \\
\cline { 2 - 5 } \multicolumn{1}{c|}{} & $\mathrm{N} .^{\circ}$ & $\%$ & $\mathrm{~N}^{\circ}$ & $\%$ \\
\hline $\begin{array}{l}\text { Total } \\
\text { Eutrofia }\end{array}$ & 1.686 & 86,91 & 1.866 & 89,89 \\
\hline $\begin{array}{l}\text { Total } \\
\text { Desnutrición }\end{array}$ & 254 & 13,09 & 210 & 10,11 \\
\hline TOTAL & 1.940 & 100,00 & 2.076 & 100,00 \\
\hline
\end{tabular}

En la Tabla 3 se observa que la población de desnutridos está en su mayor proporción constituida por niños alimentados en forma artificial, $77.5 \%$ (1976) y $63.8 \%$ (1977). Los alimentados en forma natural son $4.72 \%$ (1976) y $8.09 \%$ (1977).

Tabla 3

\begin{tabular}{|c|c|c|c|cc|}
\cline { 2 - 6 } & Total Desnutridos & \multicolumn{2}{|c|}{$\begin{array}{c}\text { Desnutridos } \\
\text { Aliment. Mat. }\end{array}$} & \multicolumn{2}{c|}{$\begin{array}{c}\text { Desnutridos } \\
\text { Aliment Art. }\end{array}$} \\
\cline { 2 - 6 } & & N. ${ }^{\circ}$ Casos & $\%$ & N. $^{\circ}$ Casos & $\%$ \\
\hline 1976 & 254 & 12 & 4.72 & 197 & 77.5 \\
1977 & 210 & 17 & 8.09 & 134 & 63.8 \\
\hline
\end{tabular}




\section{CONCLUSIONES}

1. Mediante el programa educativo se obtiene un aumento del $17.6 \%$ de la lactancia materna en 1977 con respecto a 1976, porcentaje que es significativamente estadístico. Este aumento de la lactancia materna se debe a una mayor cobertura y a una prolongación de la lactancia materna.

2. Paralelamente al incremento de la lactancia materna se observa una disminución de la desnutrición de un $2.93 \%$, que también es estadísticamente significativo.

3. Los desnutridos se distribuyen según tipo de alimentación en una configuración semejante en 1976 y 1977. Es importante el predominio de los niños alimentados en forma artificial dentro del universo de desnutridos. Conviene resaltar que no se observan desnutridos graves dentro de la población alimentada al pecho.

\section{COMENTARIO}

Cabe resaltar la importancia de la educación como instrumento de cambio de actitud en una comunidad. La educación alcanza el cumpli- miento de sus metas si se cuenta con la participación de un equipo multiprofesional que actúa en forma permanente frente al niño-madre con criterios operacionales uniformes. Todo esfuerzo realizado en pro de la lactancia materna es aconsejable, dada su favorable repercusión tanto en el individuo y la familia como la sociedad.

\section{REFERENCIAS}

1 Jones K., Tomasello E., Moya H. et al. Frecuencia de la lactancia matema según edad y estado nutritivo. Rev. Chil. Pediatr. 47(4): 346-349, julio-agosto 1976.

${ }^{2}$ Nichols B.L., Nichols V.N. Bases biológicas de la lactancia. Pediatr. 21(4): 347-355, octubre- diciembre 1978.

3 Patri A., Sepuilveda H. Evolución de la lactancia matema' Pediatr. 21(4): 281-285, octubre-diciembre 1978.

4 Zañartu J. Lactancia materna y nutrición infantil. Rev. Méd. de Chile 102(11): 882-886, noviembre 1974.

5 Valiente S., Gallardo B., Boj M.T., Urteaga C. Nutrición como factor de salud. Bol. Hosp. S. J. de Dios 18(2): 124-131, marzo-abril 1971.

${ }^{6}$ Escobar $F$. Cambios de actitud de la comunidad a través de las campañas de información. Bol. Of. Sanitaria Panamericana 82(2): 112-119, febrero 1977.

${ }^{7}$ Hambraeus L. Proprietary milk versus human breast milk in infant feeding: a critical appraisal from the nutritional point of view. Pediatr. Clin. North Am, 24(1): 17-36, febrero 1977. 